\title{
Radiosensitizer RRx-001
}

National Cancer Institute

\section{Source}

National Cancer Institute. Radiosensitizer RRx-001. NCI Thesaurus. Code C97130.

A dinitroazetidine derivative with potential radiosensitizing activity. Upon administration, RRx-001 is able to dilate blood vessels, thereby increasing tumor blood flow and thus improving oxygenation to the tumor site. By increasing oxygen levels, these tumor cells may be more susceptible to radiation therapy. Tumor hypoxia is correlated with tumor aggressiveness, metastasis and resistance to radiotherapy. 